\title{
La Pedagogía Activa en los Claustros de la Universidad de Cartagena, la Presencia del Pedagogo Alemán CARL Glockner*
}

Por: Dora Piñeres de la Ossa

\section{RESUMEN}

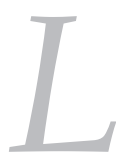

a presencia del pedagogo alemán Carl Glockner como rector de la Universidad de Cartagena y director de la Facultad de Filosofía y Letras o Colegio de Bachillerato en 1928, marcó un hito en la historia de la institución, principalmente expresado en la adecuación de las aulas, en la dotación de laboratorios y ayudas didácticas y en los inicios del seminario como estrategia pedagógica. La época de oro como algunos la definieron, marcó profundamente las mentes de los alumnos que tuvieron la experiencia formativa del pedagogo alemán.

\section{Presentación}

La historiografía sobre reformas universitarias en Latinoamérica, permite ver una mayor preocupación por el análisis e influencia de la Pedagogía Activa en las escuelas primarias, secundarias y de institutos para la formación de educadores y una escasa documentación acerca de la influencia de esta pedagogía en los estudios universitarios. ${ }^{1}$ En este artículo se intentará analizar cómo se expresó la pedagogía moderna activa en el plano de las prácticas pedagógicas, particularmente en la Universidad de Cartagena durante los años de 1.928 a 1.930 .

En Colombia la influencia de una de las reformas pedagógicas más importantes del siglo XX en el mundo occidental, no solo se dio a nivel de los estudios primarios, secundarios y de las escuelas normales, sino que también se hizo sentir en los estudios universitarios. Los principios fundamentales de la pedagogía activa de Kerchensteiner y Dewey basados en una nueva propuesta de la relación entre educación y sociedad, fueron asimilados por los discursos modernizantes que se plantearon entre los años veinte y cuarenta, que pretendieron reformar la educación universitaria colombiana en respuesta a las necesidades de la economía
* Ponencia presentada en el Segundo Simposio sobre Pensamiento P e d a g ó g i c o Latinoamericano Rud e colombia , celebrado en la Universidad de Cartagena los días 8 y 9 de Octubre de 2003

1 En el proceso de i $n$ d a g a c i ó $n$ encontramos en este sentido el artículo de Diana Dumar sobre la influencia de la Escuela Nueva y en especial del método de Decroly en las escuelas experimentales de Progreso y las Piedras, en el Uruguay, "La Escuela Nueva en el Uruguay (1.924-

1.930)" incluido en la "Historia de la Educación Latinoamericana. De la Colonia a nuestros días", Rudecolombia, Diana Soto Arango, Editora, Colección Eventos, 2.000 , Tunja. Y ver también en este mismo libro el artículo de Jaime Caicedo Escudero sobre la influencia de la Pedagogía Activa de Dewey en la educación secundaria de Chile, "La Escuela Nueva y Activa en América Latina, Influencia de Dewey en las Reformas Educacionales de Chile, $1.927-1.945 "$ ". Igualmente, Javier Ocampo López, en su texto "Colombia en sus Ideas, 1.999, Tomo III, V parte, editado por Universidad Central en Bogotá, en el capítulo La modernización en 
industrial y de la demanda de los nuevos grupos sociales que empezaban a crecer, como efecto de la movilidad estructural impuesta por el nuevo orden social. La universidad debía cumplir con la función de formar los cuadros directivos del país en los aspectos tecnocráticos, burocráticos, científicos y tecnológicos en aras del fortalecimiento de la nación.

A Georg Kerchensteiner, nacido en Munich (1.854-1.932) se le conoce como el promotor de la Escuela Profesional y la Escuela Activa. La finalidad de su pedagogía consistió en utilizar los intereses prácticos de la juventud como "punto

Colombia analiza la influencia de la Educación Nueva en Hispanoamérica en los niveles primario, secundario $y$ en las escuelas Normales. Los historiadores en Pedagogía Javier Sáenz, Oscar Saldarriaga y Armando Ospina en su texto "Mirar la Infancia: Pedagogía, Moral y Modernidad en Colombia, 1.903-1.946", analiza la presencia de lá pedagogía activa en los niveles primarios $y$ secundarios de la Educación colombiana, así como también en las Escuelas Normales y muy someramente en el nivel universitario; y por último, el texto de Ingrid Müller de Cevallos, "Temas Escogidos de la Pedagogía Alemana Contemporánea", que trata sobre los fundamentos de la Pedagogía Activa de Kerchensteiner en Alemania y sus implicaciones en la Segunda Misión Pedagógica Alemana.

2 SABIOZ Raimond. "Georg Kerchensteiner" 1.959 En los Grandes Pedagogos, Fondo de Cultura Económica. Compilador Jean Chateau. México. Páginas 215-245

${ }^{3}$ SAENZ, Saldarriaga y Ospina. Op.Cit., Página 166-167 Tomo II

4 KERCHENSTEINER, George (1.908) Citado Ingrid Müller, Op.Cit., En la Lucha por la Cultura... I Parte, página 30-31 de partida de la educación" y procurar establecer un lazo estrecho entre la enseñanza teórica y los ejercicios prácticos. Para Kerchensteiner la acción pedagógica requería la integración de tres factores: el objeto pedagógico (el alumno), los medios (su medio cultural), y el sujeto (educador).

Para él, al alumno se le debía desarrollar una personalidad autónoma a partir de sus intereses tanto inmediatos como mediatos. En relación a lo cultural se refería a la integración de los valores espirituales del hombre con la comunidad, de manera que el último fin de la enseñanza no fuera la pura transmisión de los conocimientos sino el desarrollo de los valores individuales y colectivos "como comunidades de trabajo", donde maestros y alumnos están unidos para realizar una obra común; y en cuanto al tercer factor, los maestros, señalaba, que no debían formar simplemente el ser espiritual del individuo, sino también proveer a la conservación y moralización de la comunidad, meta que solo se alcanzaría por medio de la formación profesional -el ejercicio del trabajo- en beneficio de la comunidad. ${ }^{2}$

En suma, su método de la Escuela de Trabajo concebía la ésta como un medio en que la persona aprendía por su propia actividad y experiencia. Se basaba en la formación del espíritu social como estrategia para la formación de la voluntad, la elaboración cultural y la defensa de individualidad del alumno a través del ejercicio de auto corrección, el autocontrol y el auto examen. En síntesis, se trataba de una propuesta de auto educación tanto intelectual como moral. ${ }^{3}$ Lo más importante de su método, el taller y el laboratorio eran fundamentales para la formación de un espíritu social fundamentado en el trabajo en equipo entre los alumnos que debían propiciar el compañerismo, la participación y la ayuda mutua esencial para la formación de un ciudadano dispuesto a trabajar para la sociedad. Las instituciones de educación debían equiparse según él con talleres y laboratorios: "los talleres escolares proveen el ámbito de experiencias para el dibujo, la enseñanza de la mecánica, la geometría y el cálculo; los laboratorios de física y química para la aplicación de métodos de investigación." 4 
Algunas experiencias relacionadas con el método de la Escuela Activa van a influir en la universidad de Cartagena, entre los años de 1.928 a 1.930, en un conjunto de transformaciones pedagógicas, implementadas por Carl Glockner durante su rectorado y como director de la Facultad de Filosofía y Letras de la Universidad de Cartagena.

La presencia del pedagogo alemán está relacionada directamente con los ideales de modernización del sistema educativo en Colombia. En 1923 por decreto 1595 del 22 de Noviembre fue contratada por el gobierno nacional durante la administración de Pedro Nel Ospina, la segunda misión pedagógica alemana, que arribó al país en 1924 para reorganizar la educación pública. Aunque este proyecto no se concretó por discrepancias entre los parlamentarios de la época, posteriormente algunos de sus integrantes asumieron la dirección de entidades educativas como fue el caso de Carl Glockner en la Universidad Cartagena, en 1928, quién había sido contratado dentro de esta misión para lo

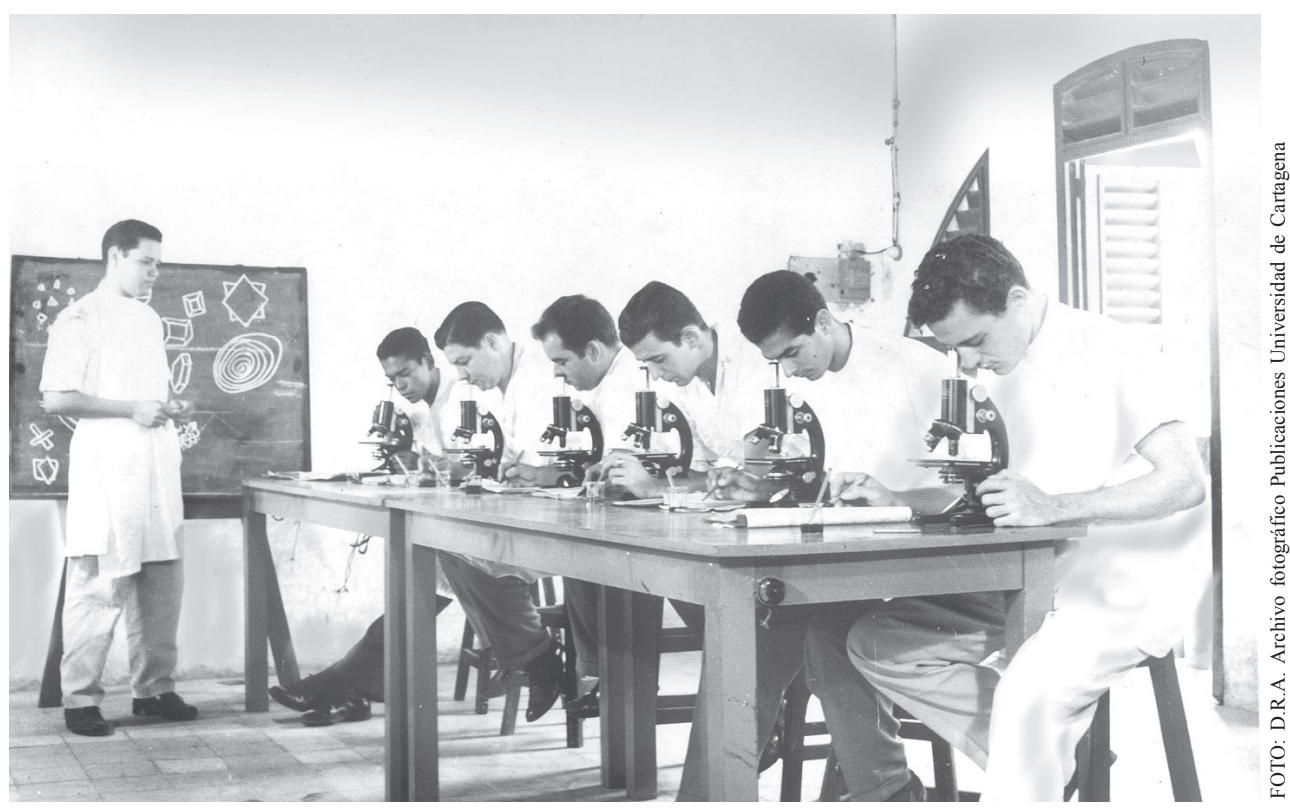
concerniente a la

reforma de la Escuela Normal, junto con Antón Eitel, para la reforma universitaria y Karl Decker para la de los colegios de secundaria. Para estos años, fueron numerosas las visitas y la inmigración de intelectuales extranjeros, como Ovidio Decroly, Raymond Buysé y Henry Pierón entre otros, los cuales marcan en la historia de la educación del país, tendencias y aplicaciones de la pedagogía activa.

Paradójicamente, aunque la función de Glockner estaba dirigida a la reforma de la Escuelas Normales, estuvo comprometido también en el proyecto de reforma del sistema educativo que propendía por la centralización universitaria en Bogota y la propuesta de supresión de las universidades departamentales, el cual fue presentado al Congreso años mas tarde por el movimiento estudiantil de la 
${ }^{5}$ Archivo Histórico de Cartagena de Indias (en adelante se citará AHC). La Patria, periódico conservador 20 de Enero de 1.928. $\mathrm{N}^{\circ} 1631$ -Página 1

${ }^{6}$ A.H.C. Ibid. 27 de Enero de 1.928 número 1637 Pág.1

${ }^{7}$ A. H. C. Ibid. Pág. 1
Universidad Nacional -aduciendo falta de recursos económicos y el escaso número de docentes que formaban de manera deficiente a los profesionales de la educación-; proyecto que, a juicio de la misión, debía entenderse como la unificación de los pénsumes de las universidades departamentales con los de la Universidad Nacional de Bogotá.

La rectoría de Carl Glockner es anunciada en la prensa cartagenera de la época, como un período importante de cambios (renovación) en la vida académica de la institución, que venía dentro de una dinámica de tradición y continuismo enmarcada en las políticas gubernamentales, en la que la universidad había sido una institución más del sistema educativo y donde la rutina en la que se enmarcaba estaba llena de privaciones y pobreza; asíg registraba el editorial del periódico conservador "La Patria" la posesión del Doctor Glockner en 1.928: "Llegó a la ciudad el ilustre institutor alemán ya conocido en Colombia, señor don Carl Glockner quien viene a encargarse de la rectoría de nuestra histórica universidad, en virtud de contrato que con él ha celebrado el gobierno del departamento. Indudablemente ésta iniciativa oficial ha sido muy feliz y merece por lo tanto, el aplauso público, aquí encontrará en lo que se refiere a éste periódico, el apoyo entusiasta a sus labores que no dudamos serán muy acertadas. ${ }^{5}$... ha llegado pues, el momento más trascendental en la vida del egregio instituto; el de la renovación completa o el de su aniquilamiento definitivo". ${ }^{6}$

Coincide la llegada del pedagogo alemán a éste centro educativo según las referencias de la misma fuente, con el aporte financiero que le hace el Congreso de la República, por medio de la Ley 11 y la Ley 45 de 1927 como subvención a la Universidad de Cartagena con motivo del primer centenario de su fundación, destinado al fomento de varias facultades a juicio del Consejo Directivo ${ }^{7}$.

Los primeros datos que se tienen sobre las reformas emprendidas por Carl Glockner en la Universidad de Cartagena son de diversa índole. Entre los de orden pedagógico figura la adopción del Método Activo de la Escuela Nueva que se vio representado con el montaje en las aulas de laboratorios de física y química y el nombramiento de profesores por concurso. Para Glockner, el interés de esta pedagogía "despertaría su actividad creativa y lo capacitaría para el trabajo productivo, disminuir el escollo en la aplicación del método por parte del profesor quien, con el derecho continuo al uso de la palabra, con sus preguntas auxiliares frecuentes, con su ayuda acostumbrada fácilmente quita al alumno la posibilidad de desplegar su propia iniciativa y descubrir algo por sí mismo".

En este mismo Informe de Glockner, señalaba que se proponía desmentir la 
creencia corriente de que "el oír con menor o mayor atención y el contestar es la verdadera actividad escolar". Para él, la aplicación del método activo en la enseñanza universitaria obligaba a un cambio en todo el proceso educativo, en el sentido de que "el profesor en lugar de exponer él mismo la materia o de dirigir la investigación colectiva estimule al alumno al trabajo espontáneo...entrenarlos al trabajo metódico, constante e inteligente... que el texto impreso no sea el único instrumento de aprendizaje en manos de los alumnos sino que este elabore su texto personal, con mapas especiales, datos estadísticos, dibujos teoremas, construcciones, resoluciones de problemas, resúmenes de experimentos y observaciones, todo

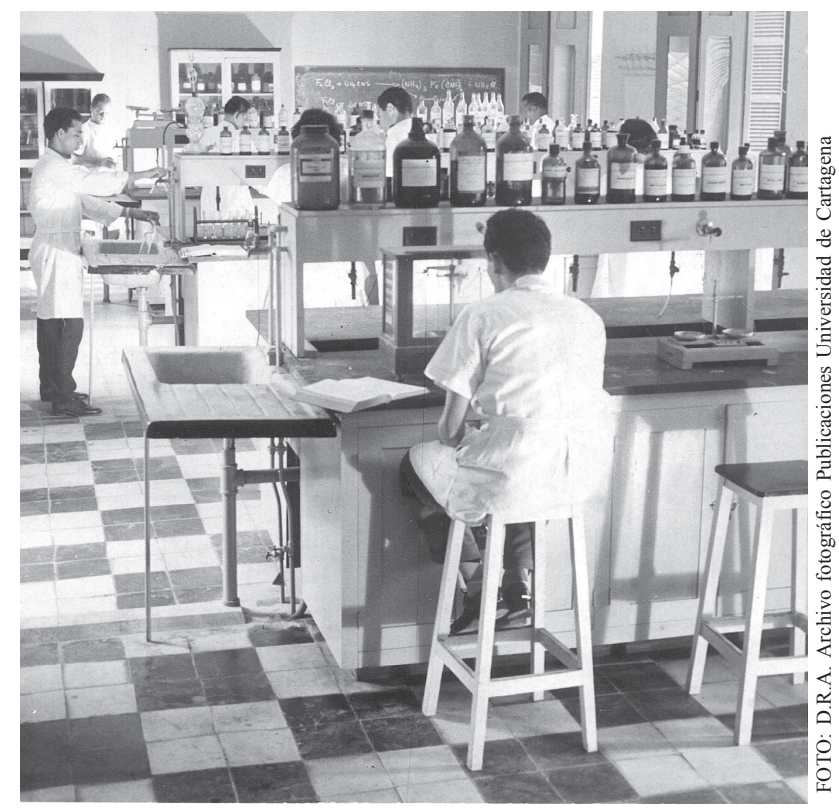
ordenado científica y pedagógicamente según el progreso diario de la clase" 8 Algunos de sus egresados dan cuenta del espíritu humanista y científico que se instituyó en la universidad ${ }^{9}$ :

Un estudiante de la Facultad de Filosofía y Letras de la época, Patricio Villalba, señala:

"Leíamos a Víctor Hugo, a Rodó, a Milton, a los literatos de la época...léamos los textos de la carrera, textos europeos sobre todo de autores italianos y franceses, textos en latín y francés...Se enseñaba a través de conferencias...nosotros tuvimos materias en francés y latín bastante dominados y la literatura española, inglesa. La historia se estudiaba con bastante profundidad... la calidad de la enseñanza de los idiomas era casi perfecta en ese tiempo, salí del bachillerato con buen dominio del ingles, del francés y del latín..."10

${ }^{8}$ AHC. Documento de 11 páginas. Informe que el rector de la universidad de Cartagena Carlos Glockner rinde al Director de Educación Pública del De p a r tamento, Cartagena diciembre 6 de 1.928

${ }^{9}$ Entrevista a Patricio

Para otros estudiantes, el énfasis del bachillerato en idiomas les dio la fundamentación para el posterior ejercicio profesional de la docencia ${ }^{11}$ :

"Salí del bachillerato con buen dominio del inglés, del francés y del latín y eso me ha servido para toda la vida... cuando terminé intenté estudiar Ingeniería Civil pero no pude terminarla por tener que trabajar, luego trabajé en dos o tres compañías norteamericanas, bancos compañías de construcción... Desde siempre trabajé por mis conocimientos de inglés, eso demuestra una vez más las ventajas de haber estudiado en la facultad de bachillerato... He sido profesor de inglés por muchos años...Y fui uno de los fundadores del Centro Colombo Americano en 1.960 entre otras..."
Villalba Verbel, estudiante de la Facultad de Filosofía y Letras de la Universidad de Cartagena entre 1.927 a 1.932
10 Entrevista a Patricio Villalba Verbel.
11 Entrevista a Lino Betancourt Villadiego, estudiante de la Facultad de Filosofía y Letras 1.931-1.938 
12 Entrevista a Tomás Figueroa Cervantes estudiante de la Facultad de Filosofía y letras 1.928-1.930

13 AHC. Diario de la Costa 10 de Enero de 1.929 Página Editorial "En los inicios del año pedagógico" y carta dirigida al director del Diario de la Costa por el Vicerrector de la Universidad Manuel peña para "que se percibiera de cerca las labores realizadas y no se juzgara anticipadamente al programa de mejoramiento" en la universidad.

14 Universidad $\begin{array}{lr}\text { Pedagógica } & \text { y } \\ \text { Tecnológica } & \text { de }\end{array}$ Colombia - (en adelante se citará UPTC)Biblioteca Fondo Posada 2336. Informe del rector Carl Glockner: Universidad de Cartagena. Mayo 10 de 1.930 Dirigido a Ministerio de Educación Nacional. En memoria del Ministro de Educación Nacional al Congreso de 1.930. Imprenta nacional 1.930. Bogotá página 116
Por otra parte, procedió al cambio de régimen disciplinario de encierro y castigo por otro más humanístico, como por ejemplo la lectura de obras clásicas y el desarrollo de la autonomía expresada en los autocontroles de salida a los alumnos internos. Igualmente, el cambio de uniformes para los estudiantes del bachillerato más acorde con el clima tropical. Para Glockner la promoción del deporte, el fortalecimiento físico a través de la gimnasia de los alumnos estuvo en los programas de atención; durante el año de 1.928 organizó un equipo de lanzamiento de disco para participar en las olimpiadas nacionales realizadas en Cali:

“Glockner era muy activo, también en el deporte logró conformar un equipo de fútbol y otro de lanzamiento de disco, en éste compitió, resultando ganador con mención especial, Sabas Bustamante Ayola estudiante de la facultad de Filosofía y Letras"12

Simultáneamente y para atender el deterioro físico del viejo claustro de San Agustín, propone su ampliación y remodelación para ponerlo a tono con las instituciones universitarias modernas, el cual fue adelantado por el arquitecto italiano Francisco Nordio:

"El histórico edificio, cuyo estado ruinoso muchos años ha venido constituyendo una vergüenza y una amenaza para todos, bien deberían visitarlo hoy quienes deseen darse cuenta de la gran obra de reconstrucción que en él se esta llevando a cabo bajo la inteligente dirección del arquitecto italiano Francisco Nordio... La reorganización del plantel y el implantamiento en éste de los métodos de enseñanza, es algo que ocupa con plausible interés la atención del señor Director de Educación Pública y del encargado de la rectoría... Los nuevos gabinetes de Física y Química en el año escolar que se inicia serán puestos al servicio de la universidad..."13

Estas primeras manifestaciones de modernización en la Universidad de Cartagena en 1.928 con la rectoría de Carl Glockner, dan cuenta de la puesta en práctica de la pedagogía activa en la institución con la presencia de laboratorios de química y física y el fomento de la investigación entre el profesorado y los estudiantes como también el hábito de suscitar la reflexión a partir de conferencias y actividades literarios sobre temas científicos de actualidad. ${ }^{14}$

El plan de estudios de la facultad de Filosofía y Letras fue modificado en el trasfondo de una reforma universitaria demandada por la autoridades universitarias a la cabeza del nuevo rector, cuya reforma buscaba modernizar los estudios que se impartían en la universidad, a partir de los asuntos pedagógicos 
basados en la Pedagogía Activa, y respondiendo en parte a la amenaza que representaba el proyecto de centralización universitaria. ${ }^{15}$

Esta reforma del plan de estudios de la facultad, también respondía a los resultados académicos deficientes arrojados a los recién reglamentados exámenes de revisión del año de 1.926, cuyas causas- señalaba Glockner- provenían de la falta de uniformidad del pensum de la Universidad de Cartagena con el de la Universidad Nacional, y del uso de la pedagogía magistral y memorística que no daba posibilidades para el desarrollo autónomo de los estudiantes ni permitía ninguna experiencia investigativa por su propia iniciativa.

${ }^{15}$ La Misión Pedagógica Alemana en 1.925 ,

La preocupación del rector alemán, que fue a la vez profesor de la misma, obedece a los motivos por los cuales fue contratado en el año de 1.923 como miembro de la segunda misión pedagógica alemana para emprender las reformas de las Escuelas Normales del país. Glockner, desde la Universidad de Cartagena, para lograr una orientación práctica de la enseñanza de la Historia y de la Geografía, adquirió para la biblioteca mapas modernos de Historia Antigua y Moderna, obras literarias y textos sobre las distintas materias y gabinetes de Física y Química. En este periodo la Gobernación del departamento otorgó becas de especialización para los estudiantes de Filosofía y Letras en universidades europeas, la especialización básicamente era en Ciencias Naturales y en Humanidades. ${ }^{16}$

Para el año de 1.929, como respuesta a esas necesidades de reforma los estudios de Filosofía y Letras empiezan a impartirse de conformidad con las disposiciones nacionales pertinentes, es decir, con unos estudios distribuidos en siete (7) años; un bachillerato técnico que comprendía cuatro (4) años de estudios y uno clásico de tres (3) años mas, para quienes desearan ingresar a facultades superiores, estos últimos tres (3) años también se le llamaba bachillerato de especialización en humanidades y ciencias exactas.

Glockner concibió a la Facultad de Filosofía y Letras como la representación del Gimnasio Humanista Alemán, de donde el rector tenía sus referencias pedagógicas dado el énfasis en las Ciencias Humanas y Exactas para la búsqueda y fomento en el estudiante de un espíritu humanista y científico, que le aportara una serie de herramientas o hábitos necesarios para su formación profesional y el ejercicio laboral, ya que a través del estudio de Idiomas, Literatura, Filosofía, Historia y las Matemáticas el alumno podía desarrollar un proceso lógico de razonamiento, exactitud y veracidad, pilares de una formación integral y asuntos importantes que le darían soporte a sus actividades laborales futuras.

redactó un proyecto de reforma en donde recomendaba entre otros aspectos, la centralización de los estudios universitarios en la capital de la República y las u n i versid a d e s regionales se convertirían en $\mathrm{f}$ a c u l t a d e s universitarias dependientes; el proyecto no se convirtió en ley por o $p \quad$ o s i c i ó $n$ parlamentaria y fue abortada la reforma, pero años mas tarde la c e n t r a li z a c i ó n universitaria volvió a ser tomado como asunto de reforma universitaria por los líderes estudiantiles del interior de país y la Universidad de Cartagena debió luchar nuevamente por su permanencia, a través de un movimiento en pro de la defensa de la universidad de Cartagena.

16 Archivo Histórico de Cartagena. (En adelante se citará AHC) Gaceta Departamental 30 de Abril de 1.928 Número 5.285 Página 2 Ordenanza Número 49 "Por la cual se conceden unos auxilios y se crean unas becas". 
17 A.H.C. (Fondo Gobernación)Libro de Ordenanzas y Resoluciones del año de 1.930. Ordenanza $\mathrm{N}^{\circ} 28$ de 1.930 .

${ }^{18}$ A.H.C. La Patria 30 de Abril de 1.930 No 2.295 .

19 A.H.C. Gaceta Departamental. 15 de Mayo de 1.930. $\mathrm{N}^{\circ}$ 5.876.Decreto $\quad N^{\circ}$ 101(Dirección de Educación Pública)

20 Se relacionan también otras reformas de carácter edilicio en el viejo Claustro de San Agustín, con la presencia del arquitecto italiano Francisco Nordio quien inicia remodelaciones en el tercer piso y la construcción del Paraninfo y de la Torre de la Universidad.
Las labores por reformar a la Universidad de Cartagena durante la rectoría de Glockner se interrumpen a principios del año de 1930 con motivo de la Ordenanza número 28 del mes de Abril, que estableció en su artículo número 6 "que para ser rector de la universidad de Cartagena, se requiere ser colombiano". ${ }^{17}$ Debido al contenido de los artículos de esta ordenanza Glockner se ve obligado a renunciar, en medio del descontento de la opinión pública y de la comunidad académica (estudiantes y profesores), ${ }^{18}$ y asume para efectos del término del contrato celebrado con la gobernación el cargo de Asesor Técnico de la Universidad y de las Escuelas Normales de la ciudad. ${ }^{19}$

Desde su nuevo cargo presiona por avanzar en proyectos que se vieron interrumpidos como la autorización al Consejo Directivo de la Facultad de Filosofía y Letras de reglamentar los exámenes orales y escritos; la emisión de la revista de la universidad publicada por la imprenta del departamento bajo la dirección del secretario de la Facultad de Filosofía y Letras, con un cuerpo de redacción formado por el rector y los profesores que este designara, en la cual se le daría publicidad a aquellos trabajos de los estudiantes que a juicio del consejo de redacción merecieran publicarse.

Después de la rectoría de Glockner, sus ideas de modernización pedagógica permanecen en el claustro universitario de Cartagena. ${ }^{20}$ Las siguientes rectorías de Raúl H. Vélez, Fernando De la Vega y José Ulises Osorio en el curso de los años de 1.930 a 1.932 en la Facultad de Filosofía y Letras, se continúa con la orientación del modelo de la pedagogía activa.

\section{BIBLIOGRAFIA}

Archivo Histórico de Cartagena de Indias. La Patria, periódico conservador $\mathrm{N}^{\circ}$ $1631,1637,5285$

KERCHENSTEINER, George (1.908). Citado Ingrid Müller., En la Lucha por la Cultura...I Parte.

SABIOZ Raimond. "Georg Kerchensteiner" 1.959 En los Grandes Pedagogos Fondo de Cultura Económica. Compilador Jean Chateau. México.

SAENZ Javier, SALDARRIAGA Oscar y OSPINA Armando. "Mirar la Infancia: Pedagogía, Moral y Modernidad en Colombia, 1.903-1.946". Tomo II 
Universidad Pedagógica y Tecnológica de Colombia. Biblioteca Fondo Posada 2336. Informe del rector Carl Glockner: Universidad de Cartagena. Mayo 10 de 1.930 Dirigido a Ministerio de Educación Nacional. En memoria del Ministro de Educación Nacional al Congreso de 1.930. Imprenta Nacional 1930. Bogotá.

\section{BIOGRAFÍA}

\section{DORA PIÑERES DE LA OSSA}

Trabajadora Social (Universidad de Cartagena), Docente de la Facultad de Ciencias Sociales y Educación de la Universidad de Cartagena, Doctora en Ciencias de la Educación, Especialista en Teorías, Métodos y Técnicas de Investigación Social (Universidad de Cartagena), Maestría en Docencia Universitaria (Universidad Pedagógica Nacional).Investigadora sobre Historia de la Universidad de Cartagena. Dirige el proyecto "La Cátedra Historia de la Universidad de Cartagena" 